\title{
Zigzag and Helical AlN Layer Prepared by Glancing Angle Deposition and Its Application as a Buffer Layer in a GaN-Based Light-Emitting Diode
}

\author{
Lung-Chien Chen, ${ }^{1}$ Ching-Ho Tien, ${ }^{1}$ Liu Xuguang, ${ }^{2}$ and Xu Bingshe ${ }^{2}$ \\ ${ }^{1}$ Department of Electro-Optical Engineering, National Taipei University of Technology, 1, Section 3, Chung-Hsiao E. Road, \\ Taipei 106, Taiwan \\ ${ }^{2}$ Key Laboratory of Interface Science and Engineering in Advanced Materials, Research Center of Advanced Materials Science and \\ Technology, Taiyuan University of Technology, 79 W. Yingze Street, Shanxi, Taiyuan, China
}

Correspondence should be addressed to Lung-Chien Chen, ocean@ntut.edu.tw

Received 30 May 2012; Accepted 5 September 2012

Academic Editor: Jinquan Wei

Copyright ( 92012 Lung-Chien Chen et al. This is an open access article distributed under the Creative Commons Attribution License, which permits unrestricted use, distribution, and reproduction in any medium, provided the original work is properly cited.

This study investigates an aluminum nitride (AlN) nanorod structure sputtered by glancing angle deposition (GLAD) and its application as a buffer layer for GaN-based light-emitting diodes (LEDs) that are fabricated on sapphire substrates. The ray tracing method is adopted with a three-dimensional model in TracePro software. Simulation results indicate that the zigzag AlN nanorod structure is an optimal buffer layer in a GaN-based LED. Furthermore, the light output power of a GaN-based LED with a zigzag AlN nanorod structure improves to as much as $28.6 \%$ at a forward current of $20 \mathrm{~mA}$ over that of the GaN-based LED with a normal AlN buffer layer.

\section{Introduction}

The numerous applications of GaN-based light-emitting diodes (LEDs) include traffic lights, back light units in liquid crystal displays, indoor and outdoor lighting, street lighting, and exterior advertising displays. As the conventional application in the recent decade, low-temperature GaN (LTGaN) buffer layers are inserted between the LED structure and substrate to increase the internal quantum efficiency of LEDs by improving the crystal quality of the LED structure [1-4]. For solid-state lighting applications, the commercial use of a patterned sapphire substrate (PSS) technology has increased the light extraction efficiency of the LED structure $[3,5-10]$. However, the LED structure on PSS requires an additional growth time and higher overhead costs than those of LED with the conventional structure.

Recently, in the structure of GaN-based LEDs, A1N has been applied between GaN epitaxial layer and sapphire substrate as a buffer layer to improve the quality of GaN epitaxial layer owing to a similar lattice constant with $\mathrm{GaN}$
[11-13]. However, glancing angle deposition (GLAD) is a highly effective means of depositing nanostructured thin films by rotating a tilted sample during deposition [14]. Factors governing the shape of nanostructured thin films include zigzags, vertical posts, helices, chiral pillars, and their optical properties, which are interesting due to birefractance [14-23]. Therefore, by extending the results of previous efforts, this study investigates the zigzag and helical AlN nanorod structures that are prepared by GLAD [24]. GaNbased LED structures are grown on a sapphire substrate with zigzag and helical AlN nanorod structures. This study also investigates how the AlN nanorod structure affects the structural and optical characteristics of the GaN-based LED with zigzag and helical AlN nanorod structures.

\section{Experimental}

Sapphire substrates were cleaned thoroughly with acetone, ethanol, and deionized water in an ultrasonic oscillator 
for $10 \mathrm{~min}$, respectively, and dried with nitrogen. A $3000 \AA$ thick aluminum nitride (AlN) nanorod structure was then deposited onto the sapphire substrate by magnetron reactive sputtering deposition at an oblique angle by using highpurity aluminum targets in nitrogen gas at a flow rate of $9 \mathrm{sccm}$ and a stable pressure of $3 \times 10^{-3}$ Torr. Next, the sapphire substrate was mounted on a rotational holder driven by a DC motor. Figure 1(a) schematically depicts the deposition vapor flux and film orientation with different angle definitions, where $\alpha$ denotes the flux arrival angle measured from the substrate normal and $\beta$ represents the columnar nanostructure inclination angle also evaluated from the substrate normal. The morphology and crosssectional structure were observed by field emission scanning electron microscopy (FESEM).

The glancing angle sputtered zigzag or helical AlN nanorod structure was incorporated into a GaN-based LED device as a buffer layer for $\mathrm{GaN}$ epitaxial growth. A $3 \mathrm{~nm}$ thick low-temperature grown GaN (LT-GaN), a $1 \mu \mathrm{m}$ thick undoped $\mathrm{GaN}(\mathrm{U}-\mathrm{GaN}$ ) layer, a $7 \mu \mathrm{m}$ thick n-type GaN:Si (n-GaN) layer, 12 pairs of undoped InGaN/GaN multiplequantum wells, a $100 \AA$ thick $\mathrm{Al}_{0.01} \mathrm{Ga}_{0.99} \mathrm{~N}: \mathrm{Mg}$ layer, and a $0.3 \mu \mathrm{m}$ thick $\mathrm{GaN}: \mathrm{Mg}$ were grown sequentially on sapphire substrates with an AlN nanorod structure as a buffer that had been prepared in advance by GLAD using metal-organic chemical vapor deposition (MOCVD). Heat treatment at $650^{\circ} \mathrm{C}$ for $10 \mathrm{~min}$ in ambient nitrogen activated the p-type dopant. LED chips were then fabricated. The surface of the p-type GaN layer was partially etched until the n-type $\mathrm{GaN}$ layer was exposed. Next, the indium tin oxide (ITO) contact layer was formed on the p-type GaN layer, and a $\mathrm{Cr} / \mathrm{Au}(50 \mathrm{~nm} / 1200 \mathrm{~nm})$ electrode was formed on the ITO contact layer and the exposed n-type GaN layer, respectively. Figure 1(b) schematically depicts the cross-section of the complete structure. Moreover, the $I-V$ characteristics were measured using a Keithley 2420 meter. Finally, the light output power of the LED lamp was evaluated using an integrated sphere detector; the measured deviation was around $10 \%$.

\section{Results and Discussion}

Figures 2(a) and 2(b) show the top and cross-sectional FESEM images of zigzag AlN nanorod structures that were deposited with oblique angles of $\alpha=80^{\circ}$ and $-80^{\circ}$. Figures 2(c) and 2(d) show the typical top-view and crosssectional view FESEM images of the helical AlN nanorod structures with one cycle. The cross-sectional images clearly demonstrate the formation of zigzag and helical nanorod structures. The top-view FESEM images reveal that the helical nanorod structure has less uniformity than the zigzag structure. The root mean square roughness of zigzag and helical AlN nanorod structures measured by atomic force microscopy is about 5.4 and $9.2 \mathrm{~nm}$, respectively. These two conditions resemble each other in the width of the nanorod structures. The measured nanorod structures inclination angles of the samples that are sputtered at $80^{\circ}$ are $37^{\circ}$. According to Figures 2(b) and 2(d), the flux arrival angle $(\alpha)$ and the columnar nanostructure inclination angle $(\beta)$ adhere to the following relationship:

$$
\beta=\xi\left[\alpha-\sin ^{-1}\left(\frac{1-\cos \alpha}{2}\right)\right] .
$$

This relationship is empirical and modified based on Tait's rule and our previous work $[25,26]$. The modification factor $\xi$ addressed by our previous work depends heavily on the deposition parameters and materials $[26,27]$.

Figure 3 presents a typical XRD pattern of zigzag and helical AlN nanorod structures deposited at various oblique angles on a sapphire substrate that has been prepared by radio-frequency magnetron sputtering. According to Figure 3(a), two dominant diffraction peaks, AlN (101) $(2 \theta=$ $\left.37.78^{\circ}\right)$ and sapphire substrate $(006)\left(2 \theta=41.72^{\circ}\right)$, were observed for the sample with normal deposition (i.e., $\alpha=$ $\left.0^{\circ}\right)$. The peak that corresponded to the AlN (101) orientation had a full width at a half maximum (FWHM) of $0.66^{\circ}$. The lattice parameters of AlN were $a=3.12 \AA$ and $c=$ $4.98 \AA$, respectively. The peak position was slightly shifted to $2 \theta=37.72^{\circ}$ when the flux arrival angle from $\alpha=0^{\circ}$ increased to $\alpha=80^{\circ}$. Similarly, for the peak position of helical AlN nanorod structures prepared by zigzag deposition $\left(\alpha=40\right.$ and $\left.80^{\circ}\right)$, the peak position was at 37.70 and $37.66^{\circ}$, respectively. This finding may be owing to a high incorporation of nitrogen into the film deposited with a high oblique angle. As is well known, AlN nanorods with a higher nitrogen content have a smaller lattice constant and degradation of crystallization corresponding to the broadening of peak width [26].

The propagation and reflection of light in the devices were examined by applying the ray tracing method associated with the three-dimensional model in TracePro software. The parameters used in the simulation were as follows: refractive index of $\mathrm{GaN}=2.54$, absorption coefficient $=100 \mathrm{~cm}^{-1}$, refractive index of AlN = 2.2, and refractive index of sapphire substrate $=1.76$. The emission rays in the active regions of all samples were set identical (100 rays) in the simulation. Figure 4 shows the ray tracing results of different structures. The light reflection ratio from the front side was $11: 9: 19: 15$ rays for the structures with normal, oblique, zigzag, and helical AlN layers, respectively. As for competition between the improvements in light reflection in the four structures, the zigzag AlN nanorod structure is the optimal buffer layer in GaN-based LED.

Figure 5 plots the light output power of the GaN-based LED with various AlN nanorod structures and a traditional GaN-based LED with GaN buffer layer. Also, the results of our previous study, the GaN-based LED with normal $(\alpha=$ $\left.0^{\circ}\right)$ and oblique $\left(\alpha=80^{\circ}\right)$ AlN structures, were demonstrated for comparison, denoted by (a) and (b), respectively. Output intensity of all LEDs initially increased linearly with an increasing injection current. The light output power of the GaN-based LED with an AlN nanoporous buffer layer at a low injection current was significantly higher than that of the GaN-based LED with GaN buffer layer, as shown in Figure 5. For instance, the value of light output power of GaN-based LEDs with normal, oblique, zigzag, and helical AlN nanorod structures, and traditional GaN-based LED was 6.22 (curve a), 5.33 (curve b), 8.0 (curve c), 6.23 (curve d), 


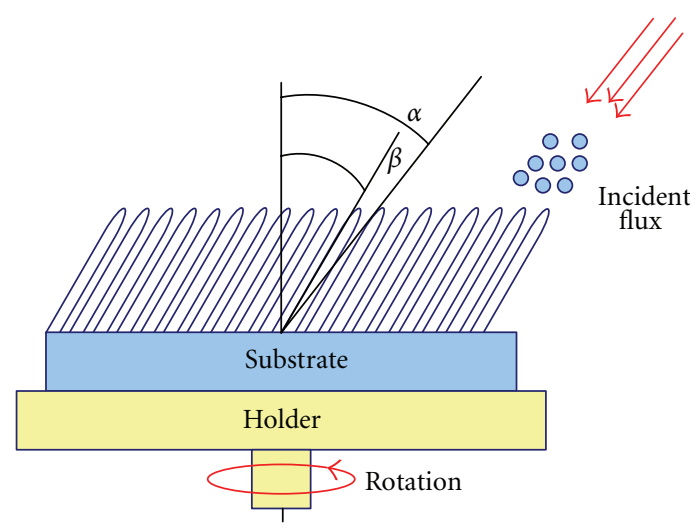

(a)

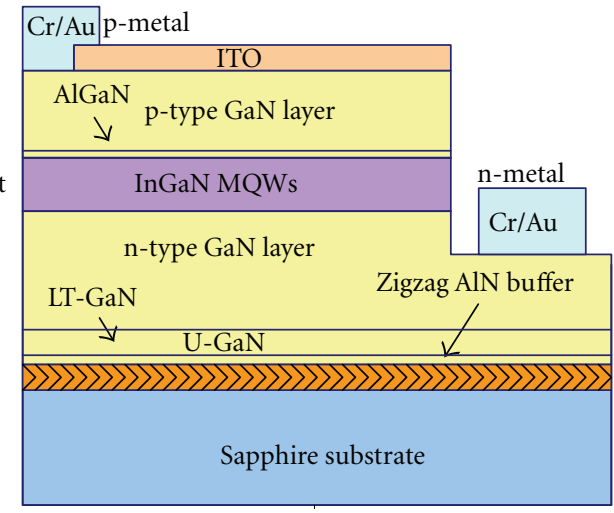

(b)

FIGURE 1: Schematic diagrams of deposition and cross-sectional view of a complete structure.

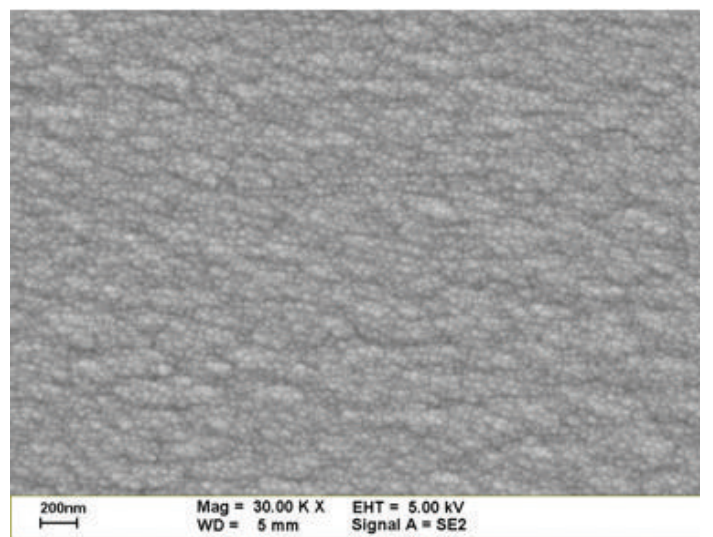

(a)

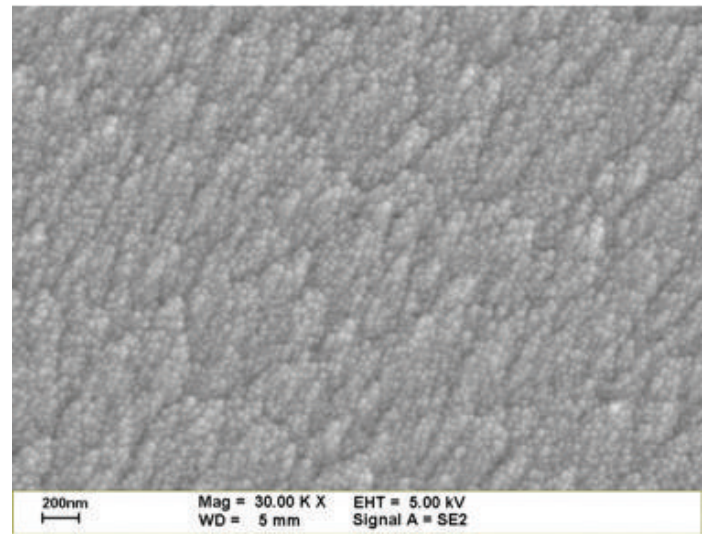

(c)

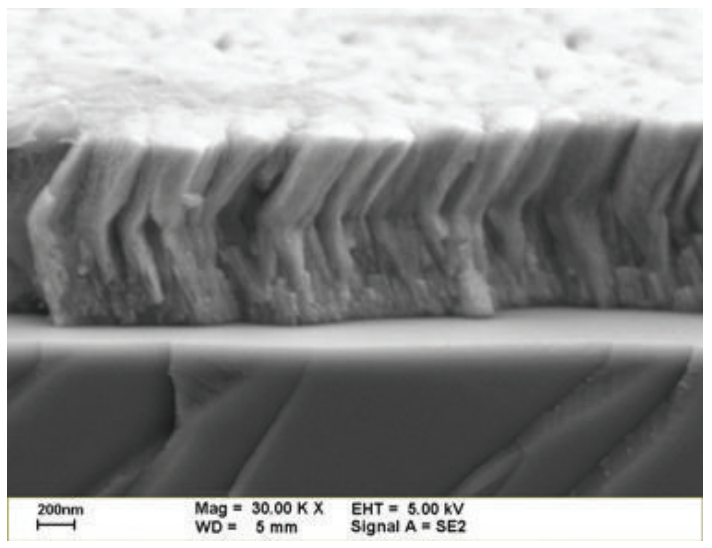

(b)

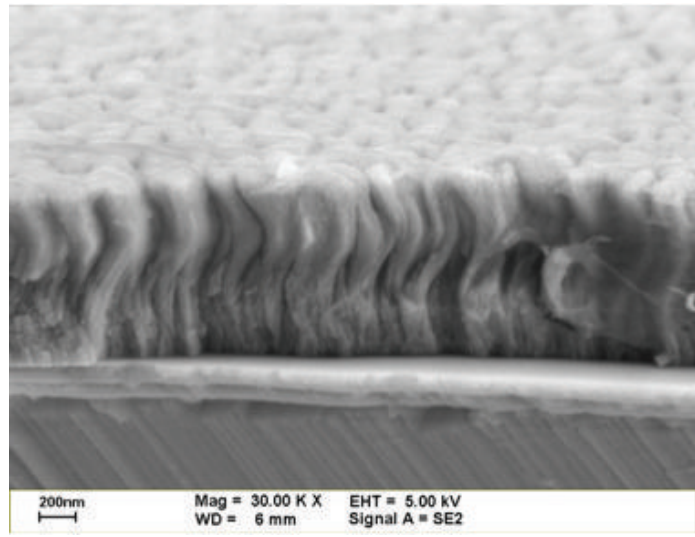

(d)

FIgURE 2: (a)-(d) FESEM images of AlN nanorod structures at zigzag and helical depositions.

and $5.18 \mathrm{~mW}$ (traditional GaN LED) at the injection current of $20 \mathrm{~mA}$, respectively. The light output power ratio was $0.78: 0.67: 1: 0.78$ for the LED structures with normal, oblique, zigzag, and helical AlN layers at an injection current of $20 \mathrm{~mA}$, respectively. The light output power of GaNbased LED with a zigzag AlN nanorod structure to the traditional LED and LED with the normal AIN structure increased by about $54.4 \%$ and $28.6 \%$ at the injection current of $20 \mathrm{~mA}$, respectively. This improvement in output power can be attributed to the scattering effect from the zigzag AlN nanorod structures, as shown in Figure 4. The ratio in light output power nearly coincides with the simulation results by TracePro software. Conversely, at a high injection current (e.g., $80 \mathrm{~mA}$ ), the light output power of LED with zigzag 


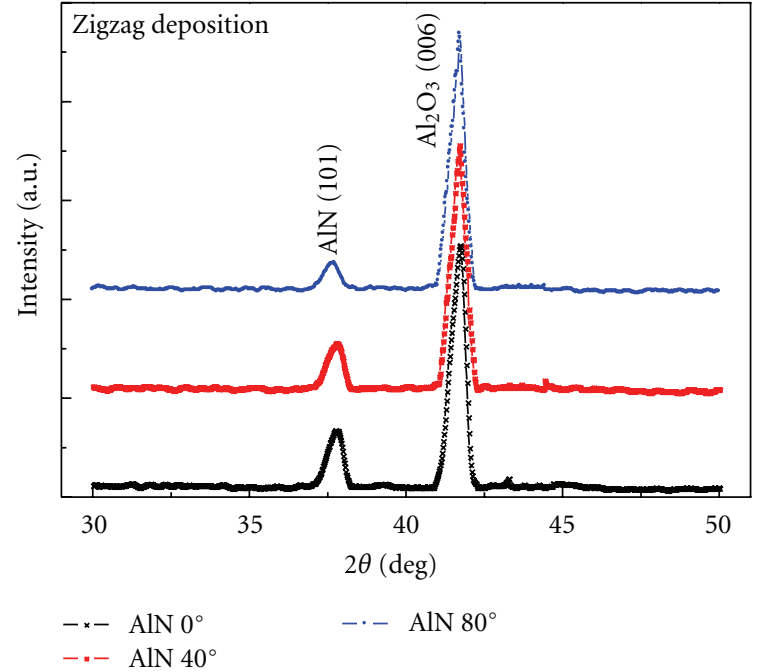

(a)

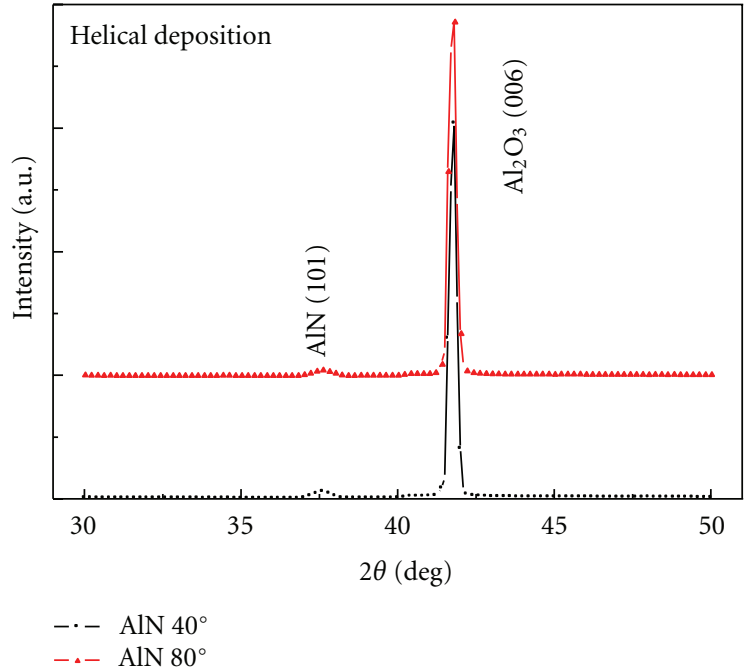

(b)

FIGURE 3: Typical XRD pattern of zigzag and helical AlN nanorod structures deposited at various oblique angles on a sapphire substrate.

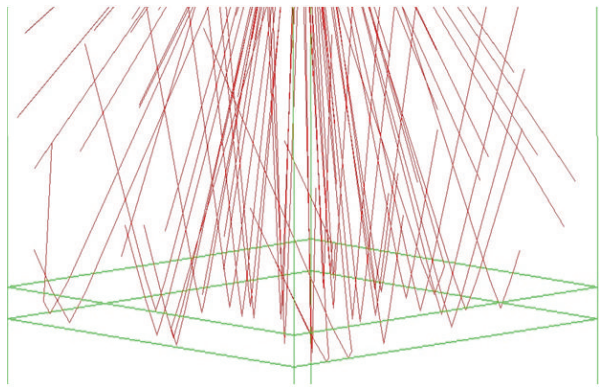

(a) $\alpha=0^{\circ}$

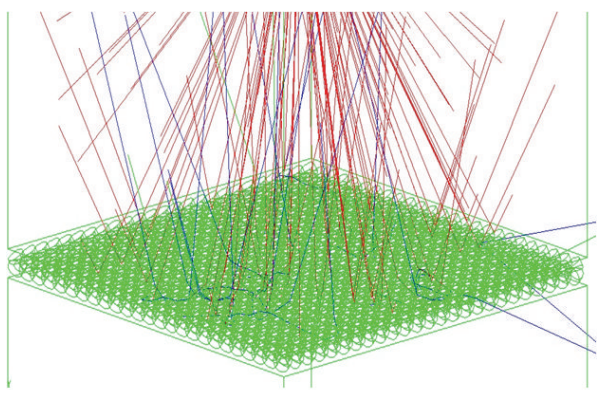

(c) Zigzag $\alpha= \pm 80^{\circ}$

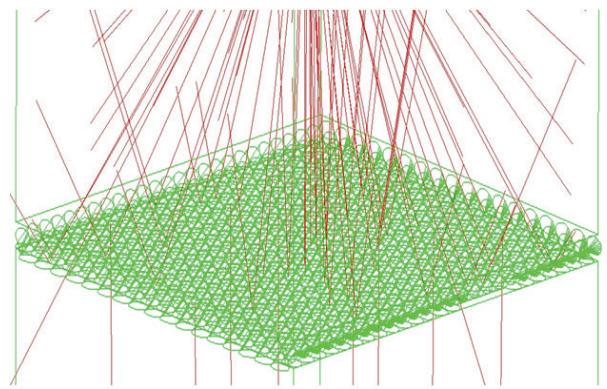

(b) Oblique $\alpha=80^{\circ}$

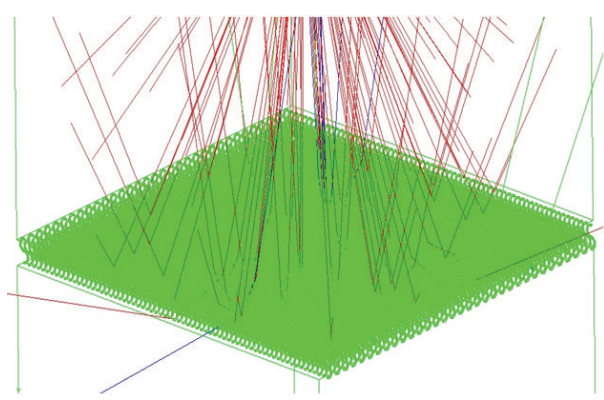

(d) Helical $\alpha=80^{\circ}$

FIgURE 4: Simulation of ray tracing for various AlN nanorod structures.

AlN nanorod structure to the traditional LED and LED with the normal AlN structure is an improvement of around $76.45 \%$ and $48.75 \%$, respectively. The thermal conductivity of AlN and GaN is 3.2 and $1.3 \mathrm{~W} / \mathrm{cm}-\mathrm{K}$, respectively [27]. Therefore, this improvement of the light output power of the LED with the AlN nanorod structures is caused by the scattering effect and a superior thermal conductivity than that of the LED with GaN buffer layer. However, the ratio in light output power slightly differs from the simulation results by TracePro software, as shown in Figure 4. This finding may be owing to the joule thermal effect as the LED operates at high injection current according to the light output powerinjection current curves in Figure 5.

\section{Conclusions}

This study investigated how the growth of GaN-based LED structures affects a sapphire substrate with an AlN 


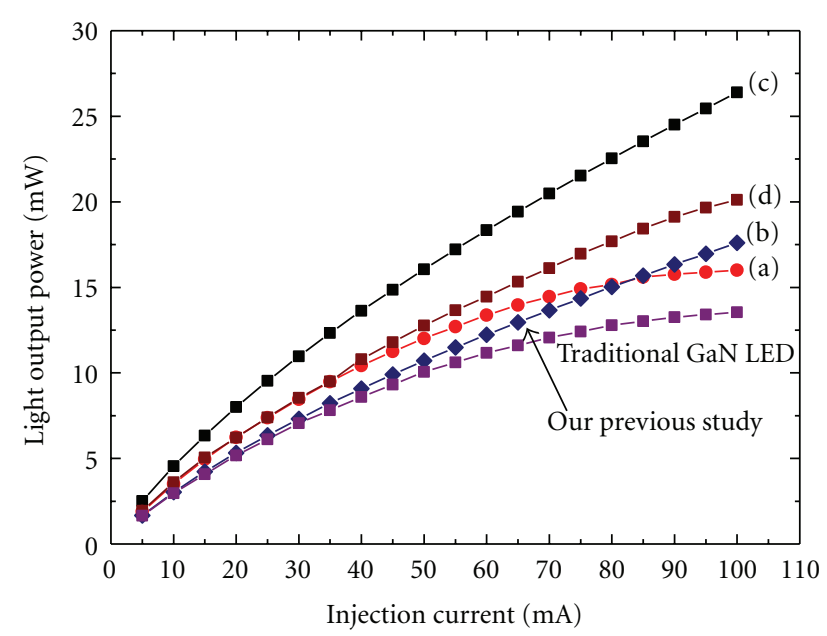

FIGURE 5: Light output power of the GaN-based LEDs with various AlN nanorod structures as a function of forward current: (a) $\alpha=0^{\circ}$ (our previous study), (b) oblique $\alpha=80^{\circ}$ (our previous study), (c) zigzag $\alpha= \pm 80^{\circ}$ (this study), and (d) helical $\alpha=80^{\circ}$ (this study).

nanocolumnar layer that was sputtered at various oblique angles. The AlN nanorod structure has a diameter of around $50-100 \mathrm{~nm}$. The ray tracing method was used with a two-dimensional model in TracePro software. The light reflection ratio from the front side was 11:9:19:15 rays for the structures with normal, oblique, zigzag, and helical AlN layers, respectively. Therefore, the zigzag AlN nanorod structure is an optimal buffer layer in GaN-based LED. In comparison with the our previous study, that is, the GaNbased LED with normal AlN buffer layer, the light output power of a GaN-based LED with a zigzag AlN nanorod structure improved to as much as about $28.6 \%$ at a forward current of $20 \mathrm{~mA}$. Furthermore, the results of light output power of the GaN-based LEDs nearly coincide with the simulation by ray tracing at a low injection current.

\section{Acknowledgment}

Financial support of this work was provided by the National Science Council of China under Contract no. NSC 100-2221E-027-015.

\section{References}

[1] S. Nakamura, "GaN growth using GaN buffer layer," Japanese Journal of Applied Physics, vol. 30, pp. L1705-L1707, 1991.

[2] R. Datta, M. J. Kappers, M. E. Vickers, J. S. Barnard, and C. J. Humphreys, "Growth and characterisation of $\mathrm{GaN}$ with reduced dislocation density," Superlattices and Microstructures, vol. 36, no. 4-6, pp. 393-401, 2004.

[3] K. Tadatomo, H. Okagawa, Y. Ohuchi et al., "High output power InGaN ultraviolet light-emitting diodes fabricated on patterned substrates using metalorganic vapor phase epitaxy," Japanese Journal of Applied Physics, vol. 40, no. 6, pp. L583L585, 2001.

[4] L. C. Chen, J. B. Huang, P. J. Cheng, and L. S. Hong, "InGaN blue light-emitting diodes with $\mathrm{ZnO}$ nucleation layers prepared by the sol-gel method," Semiconductor Science and Technology, vol. 22, no. 10, pp. 1178-1182, 2007.

[5] K. Bao, X. N. Kang, B. Zhang et al., "Improvement of light extraction from GaN-based thin-film light-emitting diodes by patterning undoped GaN using modified laser lift-off," Applied Physics Letters, vol. 92, no. 14, Article ID 141104, 3 pages, 2008.

[6] W. K. Wang, D. S. Wuu, S. H. Lin, S. Y. Huang, K. S. Wen, and R. H. Horng, "Growth and characterization of InGaNbased light-emitting diodes on patterned sapphire substrates," Journal of Physics and Chemistry of Solids, vol. 69, no. 2-3, pp. 714-718, 2008.

[7] D. S. Wuu, W. K. Wang, W. C. Shih et al., "Enhanced output power of near-ultraviolet InGaN-GaN LEDs grown on patterned sapphire substrates," IEEE Photonics Technology Letters, vol. 17, no. 2, pp. 288-290, 2005.

[8] X. L. Fang, Y. Q. Wang, H. Meidia, and S. Mahajan, "Reduction of threading dislocations in GaN layers using in situ deposited silicon nitride masks on AlN and GaN nucleation layers," Applied Physics Letters, vol. 84, no. 4, p. 484, 2004.

[9] T. Wernicke, U. Zeimer, M. Herms, M. Weyers, M. Kneissl, and G. Irmer, "Microstructure of $\alpha$-plane (2110) GaN ELOG stripe patterns with different in-plane orientation," Journal of Materials Science: Materials in Electronics, vol. 19, pp. S46-S50, 2008.

[10] B. Beaumont, P. Gibart, M. Vaille, S. Haffouz, G. Nataf, and A. Bouillé, "Lateral overgrowth of $\mathrm{GaN}$ on patterned GaN/sapphire substrate via selective metal organic vapour phase epitaxy: a route to produce self supported GaN substrates," Journal of Crystal Growth, vol. 189-190, pp. 97$102,1998$.

[11] H. Amano, N. Sawaki, I. Akasaki, and Y. Toyoda, "Metalorganic vapor phase epitaxial growth of a high quality GaN film using an AlN buffer layer," Applied Physics Letters, vol. 48, no. 5, p. 353, 1986.

[12] J. C. Zhang, D. G. Zhao, J. F. Wang et al., "The influence of AlN buffer layer thickness on the properties of GaN epilayer," Journal of Crystal Growth, vol. 268, no. 1-2, pp. 24-29, 2004.

[13] L. C. Chen, C. K. Wang, J. B. Huang, and L. S. Hong, "A nanoporous AlN layer patterned by anodic aluminum oxide and its application as a buffer layer in a GaN-based lightemitting diode," Nanotechnology, vol. 20, no. 8, Article ID 085303, 2009.

[14] M. M. Hawkeye and M. J. Brett, "Glancing angle deposition: fabrication, properties, and applications of micro- and nanostructured thin films," Journal of Vacuum Science \& Technology A, vol. 25, no. 5, pp. 1317-1335, 2007.

[15] J. G. Van Dijken, M. D. Fleischauer, and M. J. Brett, "Controlled nanostructuring of $\mathrm{CuPc}$ thin films via glancing angle deposition for idealized organic photovoltaic architectures," Journal of Materials Chemistry, vol. 21, no. 4, pp. 1013-1019, 2011.

[16] Z. Xie, B. M. Henry, K. R. Kirov et al., "Study of the effect of changing the microstructure of titania layers on composite solar cell performance," Thin Solid Films, vol. 511-512, pp. 523-528, 2006.

[17] M. J. Brett, M. W. Seto, J. C. Sit, K. D. Harris, D. Vick, and K. Robbie, "Glancing angle deposition: recent research results," in Engineered Nanostructural Films and Materials, vol. 3790 of Proceedings of SPIE, pp. 114-118, July 1999.

[18] Y. J. Park, K. M. A. Sobahan, and C. K. Hwangbo, "Optical and structural properties of a circular polarization handedness inverter prepared by using glancing angle deposition," Journal of the Korean Physical Society, vol. 55, no. 3, pp. 1263-1266, 2009. 
[19] M. M. Hawkeye, R. Joseph, J. C. Sit, and M. J. Brett, "Coupled defects in one-dimensional photonic crystal films fabricated with glancing angle deposition," Optics Express, vol. 18, no. 12, pp. 13220-13226, 2010.

[20] P. C. P. Hrudey, M. Taschuk, Y. Y. Tsui, R. Fedosejevs, and M. J. Brett, "Optical properties of porous nanostructured $\mathrm{Y}_{2} \mathrm{O}_{3}$ : Eu thin films," Journal of Vacuum Science and Technology A, vol. 23, no. 4, pp. 856-861, 2005.

[21] K. M. Krause and M. J. Brett, "Spatially graded nanostructured chiral films as tunable circular polarizers," Advanced Functional Materials, vol. 18, no. 20, pp. 3111-3118, 2008.

[22] A. Bonakdarpour, M. D. Fleischauer, M. J. Brett, and J. R. Dahn, "Columnar support structures for oxygen reduction electrocatalysts prepared by glancing angle deposition," Applied Catalysis A, vol. 349, no. 1-2, pp. 110-115, 2008.

[23] W. J. Khudhayer, N. N. Kariuki, X. Wang, D. J. Myers, A. U. Shaikh, and T. Karabacak, "Oxygen reduction reaction electrocatalytic activity of glancing angle deposited platinum nanorod arrays," Journal of The Electrochemical Society, vol. 158, no. 8, pp. B1029-B1041, 2011.

[24] L. C. Chen, C. H. Tien, W. C. Liao, and Y. M. Luo, "Obliqueangle sputtered AlN nanocolumnar layer as a buffer layer in GaN-based LED," Journal of Luminescence, vol. 131, no. 6, pp. 1234-1238, 2011

[25] R. N. Tait, T. Smy, and M. J. Brett, "Modelling and characterization of columnar growth in evaporated films," Thin Solid Films, vol. 226, no. 2, pp. 196-201, 1993.

[26] L. C. Chen, C. C. Chen, Y. T. Sung, and Y. Y. Hsu, "Obliqueangle sputtering effects on characteristics of nanocolumnar structure anisotropic indium tin oxide films," Journal of the Electrochemical Society, vol. 156, no. 6, pp. H471-H474, 2009.

[27] H. Morkoç, Nitride Semiconductors and Devices, Springer, Berlin, Germany, 1999. 

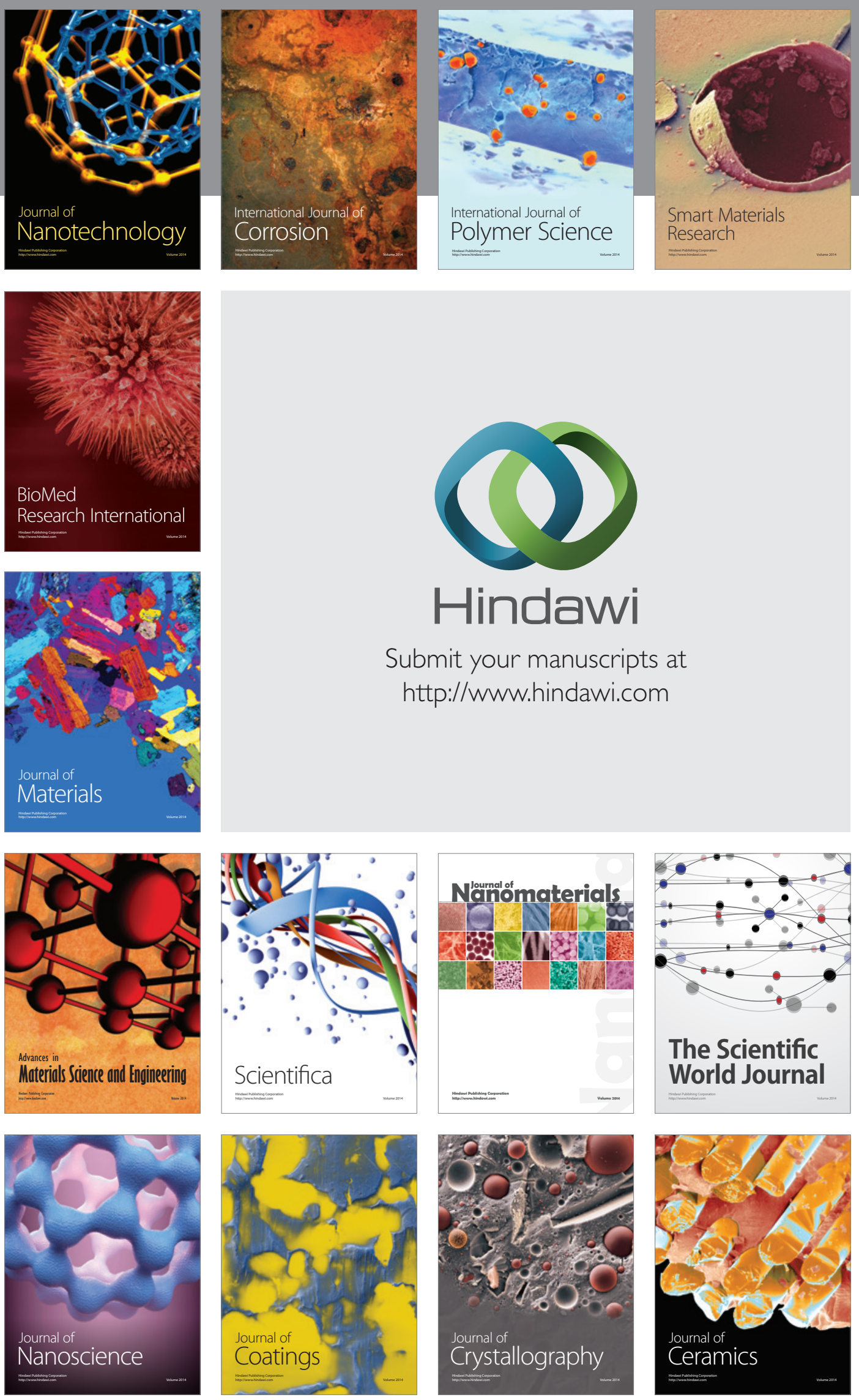

The Scientific World Journal

Submit your manuscripts at

http://www.hindawi.com

\section{World Journal}

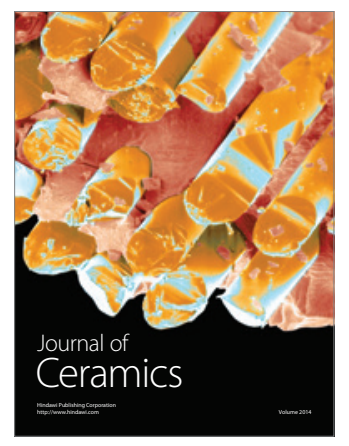

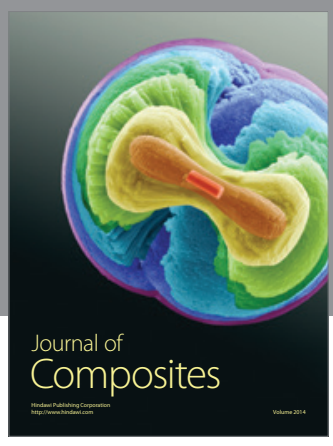
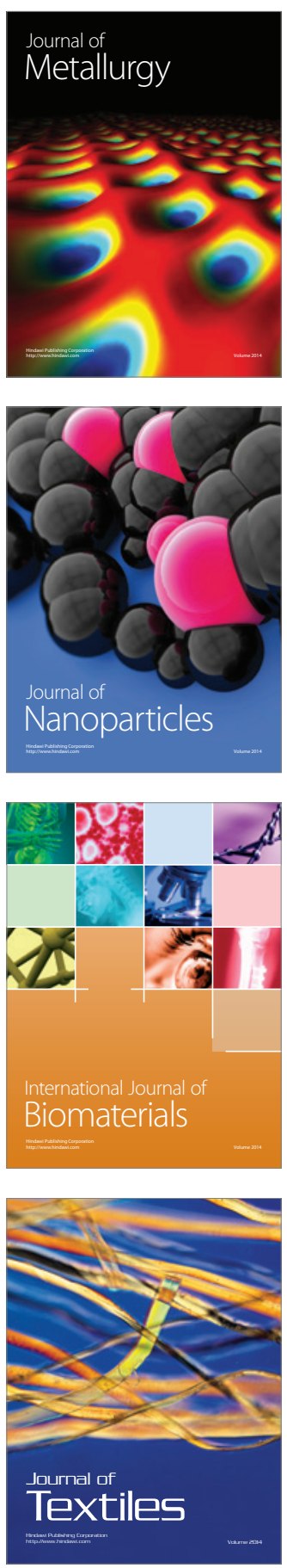